\title{
EL FINAL DE LA VIDA: SITUACIONES CLÍNICAS Y CUESTIONAMIENTOS ÉTICOS
}

\section{Gustavo G. De Simone}

Médico Especialista en Oncología Clínica, Universidad de Buenos Aires. Diploma en Medicina Paliativa, University of Wales College of Medicine. UK. Coordinador de la Sección Medicina Paliativa del Hospital "Dr. Bonorino Udaondo".

Director del Centro de Estudios PALLIUM en Medicina y Cuidados Paliativos

Director de la Carrera de Médico Especialista en Oncología Clínica, Universidad del Salvador, Buenos Aires

Correspondencia: Dr. Gustavo G. De Simone, Centro de Estudios PALLIUM.

Buenos Aires, Argentina

CC 33 Suc. 35-B (1435) Ciudad Autónoma de Buenos Aires. Argentina

$\mathrm{Tel} / \mathrm{Fax}+54$ (11) 43067608

pallium@ibm.net 



\title{
EL FINAL DE LA VIDA: SITUACIONES CLÍNICAS Y CUESTIONAMIENTOS ÉTICOS
}

\author{
Gustavo G. De Simone
}

\section{Resumen}

Los Cuidados Paliativos surgen como una modalidad de asistencia para personas que padecen enfermedades en etapa avanzada, con el objetivo de responder a sus necesidades biológicas, psicosociales y espirituales y orientar y apoyar a sus familiares. La asistencia en el final de la vida presenta determinadas necesidades y problemas, requiriendo la implementación de conocimientos específicos y la aplicación de recursos apropiados. Tanto los pacientes como sus familiares, y también el equipo profesional y las instituciones, configuran los protagonistas esenciales de situaciones donde el sufrimiento aparece como temática central. Sólo una aproximación amplia al problema, que incluya no sólo la aptitud científica requerida sino también una actitud profundamente humanitaria, permitirá encontrar respuestas a la altura de la dignidad del hombre que sufre, tanto en el aspecto técnico como en los dilemas éticos subyacentes. La referencia a un caso clínico y la reflexión pertinente pretende facilitar la comprensión global de este desafío.

PALABRAS CLAVE: Cuidados Paliativos; Proceso salud-enfermedad; Dolor; Comunicación; Futilidad médica; Necesidad médica.

\section{Resumo}

Os cuidados paliativos surgiram como modalidade de assistência para pessoas que sofrem enfermidades em fases avançadas, com a finalidade de atender suas necessidades bio-psico-espirituais, assim como orientar e apoiar seus familiares. Os cuidados no final da vida apresentam determinadas características que exigem a implementação de conhecimentos específicos e aplicação de recursos apropriados. Pacientes e seus familiares, bem como instituições e as equipes profissionais, apresentam-se como protagonistas especiais em situações onde o sofrimento é a temática central. Somente uma avaliação ampla do problema, que inclua além da qualificação científica, a necessária postura humanitária, permitirá encontrar respostas que contemplem a dignidade do ser humano que sofre. Fundamental, portanto, considerar os aspectos técnicos, assim como, os dilemas éticos subjacentes. Apresenta-se um caso clínico acompanhado de reflexão ética que visa facilitar a compreensão desse desafio. 


\begin{abstract}
Palliative Care emerges as a modality of assistance for terminally ill patients, aiming at responding to their biological, psychosocial and spiritual needs, as well as to train and give support to their families. End-of-life assistance poses specific necessities and problems that require the implementation of specific knowledge and appropriate use of resources. Patients and their families, in addition to home care professional team and agencies constitute the main protagonists of situations where suffering appears as a central issue. Only a comprehensive approach to the problem, including scientific qualification and a deeply humane attitude will permit to find responses in accordance with the suffering being's dignity. Therefore, it appears as fundamental the consideration of technical aspects and underlying ethical dilemmas. Reference is made to a clinical case and resulting ethical reflections in order to facilitate the global understanding of this challenge.
\end{abstract}

KEY-WORDS: Palliative Care; Healthdisease process; Pain; Communication; Medical futility; Medical necessity.

\section{Résumé}

Les soins palliatifs surgissent comme une modalité d'assistance des personnes que subissent des maladies dans un stade avancé, avec l'objectif de répondre à leurs besoins biologiques, psychosociaux et spirituels et d'orientation et soutient à leurs familles. L'assistance des malades en fin de vie explicite des besoins et des problèmes déterminés, en requérant la mise en place des conaissances spécifiques et l'application de ressources appropiés. En tennant la souffrance comme thématique central, autant les patients, les familles, l'équipe professionnel comme les intitutions deviennent des protagonistes essentiels. Seul un approche élargie du problème, ce qu'y inclut une attitude scientifique adéquate et une attitude profondément humaine, nous permettront de trouver des réponses à la hauter de la dignité de l'homme souffrant, tant dans les aspects techniques comme dans les dilemmes éthiques sous-entendus. La référence et la réflexion pertinente d'un cas clinique essaient de faciliter la compréhension globale de ce défi.

MOTS CLÉTS: Soins palliatifs; Procès santé-maladie; Douleur; Communication; Futilité médicale; Besoin médicale. 


\section{Introducción}

Los Cuidados Paliativos fueron definidos como "la atención activa y completa de los pacientes cuya enfermedad no responde al tratamiento curativo" (1). Su objeto es facilitar al paciente y a su familia la mejor calidad de vida posible (2). Los principios fundamentales que sustentan esta modalidad son:

- Reafirmar la importancia de la vida, considerando a la muerte como un proceso natural;

- Establecer una estrategia que no acelere la llegada de la muerte ni tampoco la posponga;

- Proporcionar alivio del dolor y de otros síntomas angustiantes;

- Integrar los aspectos psicológicos y espirituales del tratamiento del paciente;

- Ofrecer un sistema de apoyo para ayudar a los pacientes a llevar una vida lo más activa posible hasta que sobrevenga la muerte;

- Extender ese apoyo a la familia, para que pueda afrontar la enfermedad del paciente y sobrellevar el período de duelo.

El análisis de estas premisas nos permite deducir que se trata de una Medicina del cuidado antes bien que de la cura. Atenta al confort y calidad de vida de los pacientes en etapa terminal, la paliación recupera una clásica proporcionalidad en el arte de curar - "curar, a veces; aliviar, a menudo; consolar, siempre" - y reactualiza con el movimiento hospice una hospitalidad más antigua que el hospital. Su filosofía se apoya en el control de síntomas y en el sanar como dimensión espiritual del proceso salud-enfermedad (3).

Podemos hablar hoy de medicina preventiva, curativa y posventiva. En este último género, al que pertenece la medicina paliativa, se incluyen todos aquellos objetivos terapéuticos orientados a la mejoría de la cali- dad de vida (y de muerte) en pacientes con enfermedades crónicas sin expectativas de curación o restitución ad integrum. La propuesta es el cuidado continuo y la rehabilitación integral (4).

En el panorama actual de las disciplinas socio-sanitarias, la medicina paliativa implica el capítulo de la ética en el final de la vida, el problema del buen morir, la muerte digna y la eutanasia. Si bien la actitud paliativa se aleja tanto de la "mistanasia" (abandono del moribundo) como de la "distanasia" (asalto tecnológico de la agonía), puede ella también incurrir en la medicalización de la muerte, vale decir, el tratamiento de ésta como un problema técnico-tanatológico, en vez de un drama íntimo y existencial. En Un mundo feliz se anticipa esa banalización de la muerte mediante el condicionamiento del morir en un Hospital de Moribundos (5). ¿Es ésta la muerte "feliz" o confortable que procura la medicina del deseo?

La adopción de un enfoque antropológico y personalizado permite considerar al sufrimiento, al dolor y a la muerte como experiencias vitales dignas de ser vividas. $\mathrm{La}$ medicina paliativa implica la adquisición de conocimientos y habilidades técnicas que permiten combatir síntomas múltiples (dolor, náuseas, dificultad respiratoria, insomnio, etc.) así como una técnica de acompañamiento que cuenta a los profesionales como actores principales, sumando una $a c$ titud humanitaria a la aptitud científica (6). Este tipo de asistencia no está proporcionado exclusivamente por médicos, sino que también incluye a otros miembros del equipo sanitario - profesionales y voluntarios apropiadamente entrenados-y conformando un equipo de trabajo.

El tradicional enfoque naturalista positivo de la enfermedad orgánica no sugiere qué hacer con las personas enfermas en etapa incurable, avanzada y progresiva; su empeño por buscar las causas anátomofisiopatológicas de las afecciones no resuelve los proble- 
mas concretos de estos individuos enfermos. Teniendo a la mano múltiples recursos técnicos para sostener o reemplazar las funciones vitales, la medicina positiva no sabe sin embargo hasta cuándo deben prolongarse esos artificios ni qué cuidados mínimos deben mantenerse, sea cual fuere el estado patológico del enfermo. Si este modelo positivista está en revisión es porque se ve forzado a reconocer que el arsenal técnico disponible no garantiza el éxito del acto médico. Es aquí donde los pacientes murientes nos sirven otra vez como piedra de toque: el conocimiento experto sobre una determinada entidad nosológica no alcanza para comprender a la totalidad de la persona que enferma, sufre y muere.

Las enfermedades terminales nos mueven hoy a la reflexión, porque las controversias desatadas respecto de qué tratamientos corresponde implementar para combatirlas no se han resuelto todavía. Las últimas décadas han sido pródigas en propuestas —alejadas de la más pura tradición hipocrática — que pretenden justificar el cese de cuidados y aun alentar abiertamente la eliminación sistemática de personas moribundas. En contraposición con ellos y acicateados por planteos extremos, un número creciente de estudiosos ha reformulado en clave bioética la antigua ética del cuidado y del acompañamiento al muriente (7). Este movimiento se manifiesta a favor de una evaluación moral de las técnicas en uso, y puede ser interpretado como cambio fundamental en la actitud general frente a las ciencias y como un componente normativo de la decisión política.

Compartimos este camino de búsqueda de mejores aplicaciones para los progresos técnicos en beneficio de toda la sociedad, tomando en consideración aspectos no meramente instrumentales y económicos, sino también sociales, culturales y filosóficos.

\section{Cuidados paliativos: la escena}

Para identificar a las diferentes personas involucradas en la situación del cuidado en el final de la vida, proponemos simplemente una visita a cualquier hospital o centro asistencial, escenario donde tradicionalmente se desarrolla la acción médica. Sin embargo, estas escenas no se contraponen con la existencia real de muchas otras situaciones de agonía que ocurren fuera del ámbito sanitario institucional (en los hogares, en las calles...) donde viven (y mueren) personas en condiciones muy variadas de asistencia, las más de las veces carentes de cuidados mínimos de confort.

\section{El paciente}

El mundo en que se mueven los "sanos" no es el mismo que internamente experimentan los pacientes en situaciones críticas. A ellos la muerte se les aparece amenazante y ante su inminencia, diversos temores comienzan a aflorar. Esos miedos a veces son negados intelectualmente pero de todas formas representan un papel activo que debe ser identificado y comprendido por el equipo tratante. La negación que temporariamente suelen ejercer los pacientes no debe inducir una negación similar al equipo tratante, pues muchas veces los pacientes no solicitan información sobre su estado y esto no significa despreocupación sino un temor por lo que se les pueda llegar a decir. En otros casos, el miedo es aceptado, convirtiéndose en angustia, tristeza o depresión.

Los moribundos se enfrentan con un proceso concreto de deterioro físico y psíquico que los pone en condición real de morir; mientras que nosotros sabemos de la muerte en tercera persona, por el solo hecho de haber visto morir a los demás. Ellos se avienen a lo desconocido, sin certeza de lo que sucederá después. Esta aprensión se conjuga con un temor al sufrimiento, que con frecuencia involucra la pérdida de la imagen corporal (8). Tales miedos gozan de cierta generalidad y se entrelazan con las características específicas de cada persona en particular: en resumen, se presentan siempre asociados a una persona concreta, en sus condiciones sociales, religiosas, culturales, etc. 
Los pacientes —en forma más o menos manifiesta — demuestran miedo ante el apagamiento de su personalidad, detestan ser una carga para los demás, se muestran recelosos con una medicación que suponen los tornará dependientes, sufren por no poder completar una tarea, temen por el futuro de su familia y por el funcionamiento de su hogar cuando ellos ya no estén. Ayudarlos a verbalizar esas molestias, inducirlos al diálogo, disipar sus aprensiones frente a los métodos terapéuticos que se implementan y, ante todo, compartir sus sensaciones, es la manera en que el equipo tratante colabora con ellos para facilitarles una aceptación y adaptación apropiada.

El dolor es también un ingrediente del mundo humano. A través de él el hombre se interroga sobre el sentido de su existencia y sobre el paso del tiempo, buscando con desesperación un orden en el universo y aquella fórmula que rige sus transformaciones. Pero es el muriente el que establece en forma muy especial su relación con el mundo de modo doloroso, rechazando uno a uno los cambios externos a que se encuentra sometido porque los interpreta como una amenaza a su integridad. Se conjuga el dolor de causa orgánica y corporal, con el sufrimiento psíquico y social. Y éstos son apenas una parte de la crisis de sentido que se abate sobre él, comprometiendo su existencia como un todo. A esta noción de dolor y sufrimiento, Saunders C. le otorga el nombre de dolor total, y son muchos los especialistas en medicina paliativa que recogen tal concepto (8-9).

Hasta hace pocos años, los médicos no contaban con normativas claras que tipificaran la escalera analgésica que correspondía aplicar con los murientes. Era tradición medicar lo menos posible porque se reconocía de antemano la inutilidad final de ese esfuerzo. Frecuentemente se recurría a la sedación del paciente para acallar su dolor, obnubilando la conciencia de un enfermo que ya se tornaba molesto hasta para su propia familia. Estas confusiones demoraron el advenimiento de una de las líneas de acción privilegiadas en el campo de los cuidados terminales: el control de los síntomas.

Con este nombre se denomina a un conjunto de estrategias que permite el alivio del dolor en más del ochenta por ciento de los enfermos, tanto como el control adecuado de otros síntomas discapacitantes. La estrategia global para el control de los síntomas abarca, genéricamente, una secuencia de etapas: evaluación de las causas y las características de cada síntoma, explicación simple tanto al paciente como a sus familiares, implementación de métodos terapéuticos farmacológicos y no farmacológicos, monitoreo de la prescripción. Estas recomendaciones generales no deben descuidar la atención a los detalles que faciliten el alivio sintomático para cada enfermo en particular (10).

La educación en temas de alivio del dolor y control de síntomas debe realizarse tanto a nivel del público en general, como de los pacientes y sus familiares. En el ámbito profesional, esta educación es crucial tanto en el pregrado como a nivel de la atención primaria y la educación de los diferentes especialistas y expertos (11). "La calidad de vida y el confort en el final de la vida pueden ser considerablemente mejorados con la implementación de una cantidad relativamente pequeña de recursos: sólo se requiere la voluntad de lograrlo" (12).

La comunicación con la persona enferma en etapa incurable es uno de los problemas cotidianos que aparecen para quienes lo acompañan, incluyendo a los familiares, amigos, vecinos... y a los profesionales tratantes. Si en una época se polarizó el cuestionamiento entre informar o no informar la verdad sobre la gravedad de la situación, hoy es posible acceder a la experiencia acumulada y los conocimientos adquiridos para fundamentar posturas y argumentar racionalmente. En primer lugar, la mayoría de los enfermos murientes (y en especial los cancerosos y sidosos) ya conocen o al menos presienten su diagnóstico y su pronóstico (13-14). También sabemos 
hoy que el prejuicio sobre la aparición de depresión severa o intentos suicidas en quienes son informados sobre su situación carece de sustento, mientras que la llamada "conspiración de silencio" que se traduce con frecuencia en un pacto para no informar al paciente, sellado entre el profesional y la familia sin el consentimiento de aquel, trae más problemas que beneficios (15-16).

Consideramos que el problema real no reside en informar o dejar de informar el diagnóstico a los pacientes que corren un riesgo de muerte cercana, sino en quién debe comunicar la noticia, de qué manera y en qué momento. Más que un dilema ético, el problema cotidiano suele ser técnico: referido a la habilidad para comunicar (17-18). La comunicación debe girar alrededor de la voluntad del paciente, y toda información ser orientada a satisfacer una demanda de acuerdo con los intereses propios del afectado. Exceder ese límite es tan nocivo como no considerarlo en absoluto y contentarse con dar explicaciones detalladas sólo cuando una intervención mutilante debe ser justificada, o cuando se teme una acción legal. Si se quiere conocer qué es lo que el paciente en verdad desea saber, no hay más remedio que escucharlo con atención, renunciando a toda otra actividad que no sea observar, recibir sus preocupaciones, intercalar alguna pregunta con el objeto de manifestarle interés. Estas entrevistas semidirigidas son un aprendizaje de la escucha y demostrarán su utilidad en el momento de ir comunicando pérdidas parciales, de repetir una y otra vez informaciones difíciles de asimilar, de brindar sin subterfugios un diagnóstico claro y sencillo, siempre que el paciente lo requiera. En forma verbal o gestual, paciente, familiar y profesional irán compartiendo niveles de información en un proceso gradual y progresivo, que por momentos requiere la explicación verbal y en otros debe privilegiar el silencio, la mirada o el apretón de manos (19-20).

La persona gravemente enferma presenta habitualmente emociones y conductas que suelen sorprender (y a veces molestar) a sus familiares, amigos y también al equipo tratante. La importancia que atribuía al desempeño de su papel en el mundo se troca en confusión; aparece enojado ante los demás; sus silencios prolongados son interpretados como hirientes... Podrá incluso desafiar al poder médico faltando a las consultas, alterando horarios establecidos para tomar la medicación, difiriendo el inicio de tratamientos o comportándose agresivamente con el solo objeto de preservar su autoimagen.

El comportamiento de los enfermos con pronóstico de muerte no es homogéneo: cada persona imprime un sello personal a su dolencia. Algunos comparten cierta visión filosófica, otros profesan una religión, los demás mantienen una determinada conducta frente a la existencia. También están quienes se rebelan contra su destino y no claudican en ningún momento, mientras otros se abandonan en brazos de la muerte sin presentar batalla. Tal diversidad no impide que, encontrándose en situación de morir, experimenten un sentimiento de pérdida irreparable. Se desencadena entonces un repertorio de reacciones o de procesos psíquicos que diferentes investigadores analizaron y tipificaron (21-22).

Los profesionales de la salud debemos identificar y comprender estas reacciones y facilitar, para cada persona, una mejor adaptación psíquica y social. Responder al enojo con enojo, juzgar peyorativamente un comportamiento determinado, alentar expectativas irrealistas, son ejemplos cotidianos de iatrogenia en la asistencia de pacientes con enfermedad terminal.

\section{Los familiares}

La enfermedad terminal afecta no sólo al individuo enfermo sino también a los miembros de su familia. La proximidad de las relaciones afectivas hace que la aparición o la presencia de una enfermedad crónica la trastorne indefectiblemente. Por otra parte, el enfermo 
suele sufrir tanto por la situación de sus familiares como por su propia enfermedad. La familia se constituye como un sistema de relaciones interpersonales que forma una unidad de funcionamiento, de modo tal que cualquier modificación que se introduce en una de sus partes repercute en todas las restantes (23).

Una estrategia apropiada para brindar cuidados paliativos considera las vivencias del enfermo y abarca al grupo familiar, identificando sus necesidades, problemas y recursos, buscando el consenso. Suele aparecer una sutil ambivalencia en la relación entre los profesionales y la familia del paciente, más marcada cuando el mismo está hospitalizado. Bajo los pretextos de la eficiencia y del reglamento se cierra el paso a la participación activa de los allegados: zonas reservadas, horarios estrictos, falta de privacidad, comodidad insuficiente, comunicación escueta en los pasillos... Pero enojo y reproches cuando un familiar no está presente en el momento mismo en que el profesional desea entregarle una receta.

La familia que atraviesa un trance de esta naturaleza no se conforma con el "déjelo en nuestras manos". Ni aun sabiendo que se hará "todo lo posible" se resignarán a asumir un papel secundario en una obra que los reclama como actores protagónicos. Y en verdad les pertenece ese derecho a estar acompañando al que muere. Diversos estudios han demostrado una tasa de morbimortalidad más alta entre los cónyuges y familiares directos que no se ocuparon ellos mismos de la atención de su pariente (24).

La disputa entre la familia y el equipo tratante sobre la tenencia del enfermo no perjudica exclusivamente a ambas partes sino principalmente al enfermo. Es cierto que no puede subordinarse el interés del paciente al de sus familiares, pero tampoco el interés de los profesionales ni el de la medicina debe anteponérsele. Una lucha por el poder entre estos estratos colabora a deteriorar el bienestar y obliga a un replanteo honesto de objetivos y estrategias.
La colaboración recíproca entre el equipo y la familia elimina situaciones tensas, competencias y pequeñas deslealtades. Al mismo tiempo proporciona un canal de comunicación fluido y un mejor conocimiento de cada familiar, elementos de suma utilidad para adaptar el caudal de información y anticipar crisis. Si el hospital clásico se muestra aún hoy ciego ante la estructura, dinámica y patología familiar es porque el modelo operativo consagrado resulta reduccionista.

Los cuidados paliativos incorporan a la familia como unidad mínima de tratamiento, extendiendo la atención al período de duelo. Una medicina de tercer grado o posventiva, destinada a rehabilitar las relaciones interpersonales una vez que el daño ya se ha producido, aparece como un desafío a los programas sociosanitarios (25).

\section{El equipo de salud}

La escena de la asistencia en el final de la vida se completa cuando médicos, enfermeras y otros profesionales de la salud nos confrontamos con los pacientes murientes y sus familias. A partir de ese momento vemos sucederse análisis y estudios sin medida, demoras en la toma de decisiones, cambios frenéticos de un especialista a otro, depresiones y euforias injustificadas. Solemos movernos entre el activismo terapéutico y el abandono, entre la desprotección institucional y la angustia existencial. Institucionalmente se tiende a sostener que los pacientes con enfermedad en estadio terminal están excluidos de todo cuidado, y esta premisa lleva a la implementación de estrategias que no responden a las necesidades concretas que presentan los pacientes. Todas estas actitudes son síntomas que indican la detención de un proceso de crecimiento interno.

Las dificultades principales han sido reducidas por algunos autores a tres grupos básicos (26). El primero reúne a aquellas que hacen difícil asumir la muerte propia como fenómeno inherente a la vida; el segundo a la 
ausencia de entrenamiento de los profesionales, teórico y práctico, para ayudar a morir; y el tercero, a la falta de experiencia personal respecto de la muerte. A estas dificultades debemos añadir las propias del trabajo en equipo, área ésta tan carenciada en los programas de formación de los profesionales.

El control de los sintomas y el acompañamiento a los pacientes y sus familias se implementan eficientemente cuando enfermeras, médicos, trabajadores sociales y psicoterapeutas asumen responsablemente esas dificultades y transitan juntos el camino hacia la superación. Con ellos se integran otros miembros con funciones complementarias (ministros de fe, nutricionistas, terapistas físicos, voluntarios, etc.). El camino de integración reconoce la necesidad simultánea de proveer el cuidado al otro y necesitar a su vez ser cuidado, en un proceso dinámico que adquiere características peculiares para cada equipo.

El desarrollo de los cuidados paliativos surge en el mundo como un intento por modificar creativa y eficientemente la actitud y la habilidad de quienes trabajan con personas murientes, aunque muchos aspectos del tratamiento paliativo son aplicables también en las fases más tempranas de la enfermedad. Su inclusión en los sistemas de salud implica un desafío para toda la sociedad, comenzando por las mismas instituciones. Sus objetivos quedarían distorsionados si se pretendiera "medicalizar la muerte", reduciendo la aproximación a la persona muriente a un conjunto de recomendaciones elaboradas por especialistas. Tal como surge de las conclusiones del informe del Proyecto Demostrativo de Cuidados Paliativos de la Organización Mundial de la Salud en la región de Cataluña, podemos afirmar que "la implementación de Cuidados Paliativos agrega valores importantes al sistema de salud, tales como la actitud hacia el cuidado integral, la promoción de la autonomía y dignidad de la persona enferma, la reintroducción de la familia, los equipos interdisciplinarios, el sistema distrital de asistencia y la satisfacción de los pacientes y sus familiares. Su existencia no es sólo un buen indicador de la eficiencia del sistema de salud sino también de la dignidad de la sociedad".

\section{"Doctor: No quiero vivir más" (El caso de la paciente Alicia)}

Alicia tiene 34 años, grandes ojos color café y cabello oscuro. Es una hermosa mujer a pesar de las consecuencias de su enfermedad. Hace un año se le diagnosticó un carcinoma de cuello uterino, en estadio localmente avanzado. Recibió radioterapia y quimioterapia en dosis completas y posteriormente fue operada. Todos estos tratamientos no lograron erradicar su enfermedad.

Alicia vive con su esposo, Héctor, y sus cuatro hijos en el gran Buenos Aires, en una modesta y linda casa de material. Los varones, de 7 y 10 años, las niñas, de 9 y 4 años. Tiene otro hijo de una relación anterior, Luis, de 16 años, que también vive con ella, aunque pasa gran parte del tiempo con sus primos, hijos de la única hermana de Alicia. Dos de los primos son drogadictos, con conducta social muy agresiva.

En el último mes el deterioro físico de Alicia ha sido notorio. Sólo se levanta de la cama un rato durante el día, no sale ya de la casa. Con mucha dificultad intenta atender a sus hijos: la limpieza y la comida para los niños le resultan tareas casi imposibles de realizar. Su esposo comparte con ella muy poco tiempo, ya que está fuera de la casa prácticamente todo el día.

Desde hace cuatro días Alicia viene soportando un dolor intensísimo, no puede dormir. Además tiene vómitos y no orinó en las últimas 24 horas. Norma, la hermana de Alicia, la convence para ir al hospital.

En la Guardia del Hospital se comprueba que presenta una obstrucción de sus vías urinarias, por lo cual un médico propone una nueva intervención quirúrgica para intentar 
extirpar parte de la enfermedad. Otro especialista opina que esa cirugía es un exceso — dado lo avanzado de su tumor-y aconseja el intento de colocación de un catéter de derivación urinaria para impedir que la obstrucción actual la lleve en horas o días a la muerte producida por insuficiencia renal.

Alicia no acepta ningún tratamiento. No habla con los médicos, excepto para decirle a uno de ellos: "Doctor, no quiero vivir más..."

Un médico de la Guardia le dice a la hermana de Alicia: "Es mejor que la lleven a la casa, no podemos hacer nada".

El escenario donde transcurrió esta historia real se tiñó no sólo con la dramática situación de la joven mujer en el final de su vida, sino también con la angustiante vivencia de sus familiares (los presentes y los ausentes) y con las diferentes argumentaciones y propuestas de los profesionales inicialmente intervinientes. Se escucharon reclamos y opiniones muy diversos, sustentando conocimientos científicos y procedimientos técnicos tanto como cuestionamientos éticos y legales:

"Debiéramos llevarla a quirófano ya, hay una vida humana en juego".

"Debemos convencer al marido: mándenlo a llamar".

"Si muere en quirófano, al menos lo intentamos".

"No podemos tenerla en casa: piense Ud. que hay niños".

“Para qué la traen al hospital si después no acepta la indicación del médico?".

"Es inútil cualquier intento, permitamos que muera en paz...".

"La eutanasia es ilegal. No nos pueden pedir eso".

"¿No es conveniente solicitar la intervención de un juez?".

"Debiéramos consultar al Comité de Ética".

La enfermera se acerca a Alicia y se sienta a su lado, junto a la cama ubicada en un extremo de la sala de Guardia. Mira a Alicia a los ojos, y le toma la mano. Los médicos estaban deliberando en la oficina; Norma había salido en busca de un teléfono público para llamar a Héctor a su trabajo.

- "Alicia: yo soy Marta, la enfermera del equipo de Cuidados Paliativos. Sé que estás con mucho dolor, quizás aceptes que te ayudemos para aliviarlo..." Las primeras palabras de Marta hacia Alicia, precedidas por el lenguaje gestual, le permitieron a la paciente, por primera vez desde mucho tiempo, sentirse comprendida.

- "No quiero ya más operaciones, no puedo más". Alicia cambia su mirada y sus ojos están brillosos.

- "Nadie va a hacer nada con tu cuerpo mientras te opongas. Me dices que no puedes más..."

- "Estoy sufriendo mucho. El dolor es tremendo... Vomito todos los remedios que me habían dado. Ya no puedo hacer nada... Hay tantos problemas..."

- "Alicia: estás con mucho dolor y no duermes desde varias noches. Tienes vómitos, estás cansada, sientes que los problemas son demasiados para tus fuerzas. Podemos ayudarte a controlar tu dolor y tus vómitos. Si lo aceptas, podemos también conversar con tu familia..."

Alicia aceptó ser evaluada por el médico del Equipo de Cuidados Paliativos, quien conjuntamente con la enfermera Marta sopesaron esta situación clínica de "dolor desbordante". En consenso con los otros profesionales y el Comité de Ética, se estimó como necesidad prioritaria el alivio del dolor, propiciando además la comunicación con el marido de Alicia. Se administró una dosis de $10 \mathrm{mg}$ de morfina y 2,5 mg de haloperidol por vía subcutánea, para el control del dolor y de la emesis secundaria al estado urémico, respectivamente.

Alicia descansó más de cuatro horas. Cuando se despertó, aliviada, se dirigió a la enfermera y, entre otros temas, le preguntó: "¿De qué se trata esa propuesta de un catéter para orinar?" 
Alicia aceptó la posibilidad de una derivación ureteral, que pudo realizarse ese mismo día exitosamente. Con los síntomas adecuadamente controlados y el consentimiento de Héctor y Norma, regresó a su hogar dos días después. El trabajo coordinado de diferentes miembros del equipo (trabajador social, enfermera, médico, ministro de fe...) permitió facilitar una comunicación más abierta entre la paciente, los familiares y los profesionales. La preocupación prioritaria de Alicia era el cuidado de los hijos, especialmente Luis. Pudieron organizar un mejor sistema de cuidados, adaptando las posibilidades y los recursos psicosociales.

El equipo continuó asistiendo a Alicia en su domicilio hasta su muerte, acaecida dos meses después, por obstrucción intestinal. En los últimos días, Alicia y su familia decidieron permanecer en el hogar. Los síntomas estaban controlados, pese al progresivo deterioro en sus fuerzas físicas. Los niños encontraron la posibilidad de manifestarse en sus sentimientos y fantasías, y Héctor pudo - con la ayuda de todos- enfrentar la situación asumiendo sus responsabilidades de esposo y padre de familia.

En encuentros posteriores con Héctor y los niños no aparecieron signos de alarma durante la etapa inicial del duelo. Se facilitó orientación especializada para el tratamiento de los adolescentes con conducta adictiva, a través de un centro comunitario de salud distrital.

\section{Algunas reflexiones éticas}

La Bioética está orientada a resolver con rectitud y mediante el entendimiento los problemas morales de toda demanda por necesidad en el cuidado de la vida (27). Este objetivo implica reconocer que la ética de los cuidados paliativos es aquélla de las ciencias médicas en general, aunque es preciso identificar cuáles son las necesidades específicas que surgen en el contexto del final de la vida.
Es aquí donde aparece una dualidad: la preservación de la vida y el alivio del sufrimiento. En el final de la vida, el alivio del sufrimiento tiene aún más importancia, en la medida que la preservación de la vida se torna progresivamente imposible, irreal. Es por ello que una estrategia terapéutica apropiada para un enfermo agudo, potencialmente recuperable, puede ser inapropiada en la etapa terminal (28).

La identificación de una demanda por necesidad significa la existencia de una argumentación verdadera que pide ser satisfecha de un modo inequívoco. La bioética se ocupa de los pedidos de satisfacción de todo aquello que impide a alguien ser libre de juzgar preferencias y ejercer la voluntad para convertirse en responsable. Es menester diferenciar este criterio de necesidad de aquél que se fundamenta en el deseo: mientras la necesidad busca el bienestar en la realidad y se satisface cuando alcanza un objetivo realista y limitado, el deseo busca el bien absoluto en fantasías inconscientes, no tiene objeto real de satisfacción ni límites a su demanda (29). La insatisfacción justificable de la necesidad conduce a la persistencia de la demanda ética o al conflicto, mientras que la insatisfacción del deseo y su conflicto inconsciente no son materia de la ética sino de la cura psicológica. De esta forma, será infructuoso pretender una normativa ética para la resolución de aquellos conflictos psíquicos, así como será deshumanizante la desatención de las emociones y manifestaciones psíquicas de los pacientes, sus familiares y los profesionales de la salud, que requieren un abordaje apropiado en el contexto psicológico.

Los cuatro principios éticos cardinales de Beauchamp y Childress deben ser aplicados sobre un criterio de fondo de respeto por la vida, pero también de aceptación de la inevitabilidad última de la muerte. En el proceso de toma de decisiones en el final de la vida se involucran tres dicotomías que deben ser consideradas en una forma balanceada (30): 
- Los beneficios potenciales del tratamiento deben ser balanceados contra los riesgos potenciales.

- La decisión de preservar la vida debe confrontarse, cuando ello se vuelve biológicamente fútil, con la provisión de confort en el proceso de morir.

- Las necesidades del individuo deben balancearse con aquéllas de la sociedad.

Deben hacerse todos los esfuerzos para fortalecer la autonomía del paciente y de la familia, a través de su participación en el proceso de toma de decisiones respecto a las terapéuticas planteadas. La explicación honesta y simple de los tratamientos permitirá a los pacientes expresar un consentimiento informado o su rechazo informado, facilitándoles una sensación realista y continua de estar respaldados, proveyéndoles consejos apropiados y ayuda práctica.

La solicitud de consentimiento o rechazo informado presupone una comunicación veraz y confidencial, y se sustenta en tres requisitos fundamentales: la competencia plena del paciente, la información veraz sobre el tratamiento, y la libertad de decisión (31). En el marco del sufrimiento en el final de la vida, es una prioridad fortalecer la libertad de decisión a través de la resolución de aquellas necesidades básicas que, estando insatisfechas, se convierten en coacción y fuente de conflicto: el alivio del dolor y los síntomas físicos que lo acompañan es una realidad frecuente que aún no recibe respuestas eficientes. La soledad, la incertidumbre, el desasosiego suelen ser parte de esta escena huérfana de alivio.

La angustiante experiencia de un paciente que solicita su muerte, o aun que comete suicidio, está habitualmente relacionada con la presencia de dolor y/o depresión psíquica no controlados. La provisión de cuidados paliativos es la respuesta apropiada de la sociedad a las preocupaciones de la gente sobre el cuidado y confort en el final de la vida, fortaleciendo la autonomía de los enfermos (32).
Los profesionales de la salud, especialmente los médicos, debemos comprender el significado de "éxito terapéutico". Se trata de alcanzar un beneficio terapéutico para el paciente considerado como un todo, y no simplemente de proveer un efecto sobre alguna parte del cuerpo. En definitiva, no se limita a una pregunta sobre "tratar o no tratar", sino sobre cuál es el tratamiento más apropiado para indicar a un paciente, basándose en su pronóstico biológico, los objetivos y efectos adversos, y las circunstancias personales y sociales del enfermo (33). La corrección de la cifra plasmática de bilirrubina, el nivel sérico de hemoglobina o de la presión arterial de oxígeno son efectos que adquieren su justificación solamente en el contexto del beneficio concreto que signifique su corrección para los intereses del paciente.

Está éticamente justificado discontinuar el uso de técnicas para prolongar la vida cuando su aplicación significa una carga o sufrimiento para el paciente, desproporcionado al beneficio esperado. La vida no es un bien absoluto: no existe una obligación de emplear tratamientos cuando su uso puede ser descripto mejor como una prolongación de la agonía. Llega un momento, en tiempo distinto para cada paciente, cuando los esfuerzos basados en la tecnología pueden interferir con los valores personales más importantes (34).

El uso de sonda nasogástrica, infusiones endovenosas, antibióticos y otras intervenciones como quimioterapia, cirugía y alimentación asistida o respiración artificial son medidas de soporte para pacientes potencialmente recuperables. El uso de estas medidas en quienes no tienen expectativas de recuperación o están cercanos a su muerte es generalmente inapropiado (34).

No existe obligación de prescribir tratamientos fútiles, entendidos como aquellos los que no tienen expectativa de proveer un beneficio terapéutico (35). El concepto de futilidad médica debe comprenderse como un criterio cualitativo, en cuanto a su impacto sobre la resolución de las necesidades fundamentales de los pacientes (calidad de vida). 
En la mayoría de los países latinoamericanos es todavía más frecuente que se ofrezca a los pacientes tratamientos con intención curativa (o para controlar la enfermedad) que cuidados paliativos. Aquellas terapias se justifican:

- Cuando la curación es médicamente posible.

- Cuando existe probabilidad demostrada de prolongar la vida significativamente.

- En el contexto de investigación clínica con nuevos métodos potencialmente efectivos, con el marco académico, ético y legal de la investigación.

El concepto de futilidad médica se confronta con el de necesidad médica: todo aquello que la medicina puede hacer para responder a lo que las personas reclaman como necesario. Este cuidado mínimo necesario abarca las acciones que han demostrado proveer beneficio significativo sobre la salud. La provisión de cuidados paliativos ha dejado en claro su pertenencia a este conjunto de medidas (36).

La implementación de terapéuticas paliativas para el control de los síntomas puede, en ocasiones, acelerar la muerte. Un tratamiento paliativo con efectos adversos significativos es ética y legalmente aceptable si el objetivo es hacer el bien, aliviando el sufrimiento (37). Esta consideración ética de rectitud moral, conocida como principio del segundo efecto, presupone la validez científica y pericia técnica en la implementación del tratamiento: no puede justificarse moralmente la sedación de un paciente a través de un "cocktel" de múltiples fármacos o en dosis excesivas, puesto que se trata de un acto de impericia médica. Del mismo modo que la corrección de la hipoglicemia en un paciente diabético implica una correcta intervención diagnóstica, fisiopatológica y terapéutica, el control de síntomas en enfermos en etapa terminal responde a criterios científicos establecidos y orientados en la búsqueda del bien.

Expertos convocados por el Centro Colaborador en Oxford de la Organización Mundial de la Salud publicaron una recomendación general, con conceptos avalados por diferentes sociedades científicas, entidades sociales y credos religiosos (incluyendo el catecismo de la Iglesia Católica): "Los cuidados paliativos son la opción recomendada cuando la enfermedad del paciente es incurable y los tratamientos agresivos para prolongar la vida pueden producir morbilidad exagerada o alto costo personal, con escaso beneficio significativo. Esta recomendación se basa no sólo en elementos científicos y morales del cuidado del enfermo, sino también en una ética social relacionada con el principio de justicia” (38).

Para afianzar decisiones más apropiadas en el cuidado en el final de la vida, será necesario que mejoremos el diálogo entre quienes desarrollan normativas éticas y quienes trabajamos al lado de la cama del enfermo, que alentemos la discusión interdisciplinaria de temas éticos, que atendamos tanto a los aspectos psicológicos como a la bioética en la toma de decisiones, que discutamos y difundamos lineamientos-guía, $y$, finalmente, que tengamos la voluntad de avanzar desde esos lineamientos hacia los cambios en las prácticas y políticas de salud. Es un desafío que involucra a toda nuestra sociedad en el inicio del tercer milenio. 


\section{Referencias}

1. European Association for Palliative Care. Newsletter 1989; 1.

2. Organización Mundial de la Salud. Alivio del dolor y tratamiento paliativo en cáncer. Informe de un Comité de Expertos. Ginebra: OMS; 1990. (Serie de Informes Técnicos 804).

3. Mainetti JA. Bioética ilustre. La Plata: Editorial Quirón; 1994.

4. Doyle D, Hanks GW, MacDonald N, (eds). Oxford Textbook of Palliative Medicine, Oxford: Oxford Medical Publications; 1993.

5. Mainetti JA. Estudios bioéticos. La Plata: Editorial Quirón; 1993.

6. Cecchetto S. Curar o cuidar, bioética en el confin de la vida humana. Buenos Aires: AdHoc; 1999.

7. Dick A. An alternative to an ethics of euthanasia. In: Williams RH. To live and die. New York: Springer Verlag; 1973.

8. Saunders C. Cuidados de la enfermedad maligna terminal. Barcelona: Salvat; 1980.

9. Lewis CS. The problem of pain. New York: MacMillan; 1978.

10. Twycross RG, Lack SA. Therapeutics in terminal cancer. 2nd ed. London: Churchill Livingstone; 1990.

11. Bonica JJ. Treatment of cancer pain: current status and future needs. In: Fields HL. (ed) Advances in pain research and therapy. New York: Raven Press; 1985 (vol 9): 589-616.

12. Stjernsward J. WHO cancer pain relief programme. In: Hanks GW, (ed). Pain and Cancer. Oxford: Oxford University Press; 1988: 195-208. (Cancer Surveys Series 7).

13. Giraudi HS, Manzini JL, Somoza. El tabú de la verdad en el cáncer: seguimiento de 30 pacientes que conocieron su diagnóstico. Quirón 1989; 20(2): 45-54.

14. Oken D. What to tell cancer patients: a study of attitudes. Journal of the American Medical Association 1961: 175.

15. Weisman A. On dying and denying. A psychiatric study of terminality. New York: Behavioral; 1972.

16. Simpson MA. Social and psychological aspects of dying. In: Wass $\mathrm{H}$ (ed). Dying facing the facts. Washington: Hemisphere; 1979: 33-36.
17. Manzini JL. Bioética paliativa. Quirón 1997; 75-94.

18. Manzini JL, Somoza EJ. Asistencia al paciente terminal. Primer Encuentro Internacional para el Cuidado de la Persona con Enfermedad Terminal; 1986; Buenos Aires, Argentina. Buenos Aires: Academia Nacional de Medicina; 1986.

19. Manzini JL. Pautas acerca de la comunicación con el paciente terminal y su familia, especialmente sobre su diagnóstico y pronóstico. Material preparado para el módulo 5to del II Curso Internacional de bioética de la ELABE, Gonnet; 1991.

20. Lichter I. Communication in cancer care. Edinburgh: Churchill-Livingstone; 1987.

21. Kubbler-Ross E. Sobre la muerte y los moribundos. Madrid: Grijalbo; 1975.

22. Gómez Sancho M. Medicina paliativa: la respuesta a una necesidad. Madrid: Arán; 1998.

23. Miller RD, Krech R and Walsh D. The role of a palliative care service. Conference in the management of the patient with advanced cancer. In: Palliative Medicine 1991; 5: 34-39.

24. Carr AT. Muerte y duelo. En: Martin H. Psicología en el trabajo social. Madrid: Pirámide; 1992: 290-291.

25. Shneidman ES. Postvention. The care of the bereaved. In: Pasnau R (ed). Consultation laison psychiatry. New York: Grune and Stratton; 1975.

26. García R, Worm I. El niño muriente. Revista del Hospital de Niños de Buenos Aires 1996; 28 (115).

27. Mainetti JA. Bioética sistemática. Buenos Aires: Editorial Quirón; 1991.

28. Hastings Center. Guidelines on the termination of life-sustaining treatment and the care of the dying. Blooming: Indiana University-Press; 1987.

29. Tealdi JC. La problematización del conocimiento médico y los orígenes de la Bioética. En: Escuela Latinoamericana de Bioética, Fundación Mainetti. IV Curso Intensivo de Bioética, La Plata; 1996: 1-3.

30. Beauchamp TL, Childress FJ. Principles of biomedical ethics. $2^{\text {nd }}$ edic. New York: Oxford University Press; 1983.

31. Zanier J. Expectativas de los pacientes, los equipos de salud y las instituciones 
El Final de la Vida: Situaciones Clínicas y Cuestionamientos Éticos - G. de Simone

asistenciales de la población marplatense. En: II Jornadas Argentinas y Latinoamericanas de Bioética, Mendoza; 1996.

32. Kung H, Jens W. Morir con dignidad, un alegato a favor de la responsabilidad. Madrid: Trotta; 1997.

33. Gracia Guillén D. Procedimientos de decisión en ética clínica. Madrid: Eudema.

34. Cassem N. When illness is judged irreversible: imperative and elective treatments. Man and medicine 1976; 2: 154-166.
35. Schneiderman LJ, Faber-Langedoen K, Jecker NS. Beyond futility to an ethic of care. American Journal of Medicine 1994; 96: 100-114.

36. Roy DJ. Ethics in palliative care. Journal of Palliative Care 1997; 3: 3-5.

37. Law Reform Commission of Canada. Euthanasia, aiding suicide and cessation of treatment. Ottawa; 1983.

38. WHO Collaborating Centre for Palliative Care. Looking forward to cancer pain relief. International consensus on the management of cancer pain. Oxford: CBC; 1997. 